\title{
Les soins infirmiers en oncologie: la recherche d'un équilibre dans un système de soins en évolution
}

\author{
par Debra Bakker, Margaret I. Fitch, Esther Green,
} Lorna Butler et Karin Olson

\section{Abrégé}

La restructuration des soins de santé a entraîné des changements importants sur le plan de la charge et du milieu de travail pour les infirmières en oncologie. De récentes études décrivent l'impact de ces changements sur l'ensemble de la main-d'œuvre infirmière de plusieurs pays, mais aucune étude publiée n'a porté sur les questions de la vie au travail chez les infirmières en oncologie du Canada. C'est pour cette raison que nous avons mené une étude qualitative afin de bien saisir la manière dont les soins infirmiers en oncologie ont changé au cours de la dernière décennie et la manière dont les infirmières en oncologie du Canada gèrent ces changements. L'analyse des entrevues téléphoniques réalisées auprès de 51 infirmières en oncologie qui exercent actuellement au pays a permis de dégager trois thèmes principaux. Le premier "Le milieu des soins de santé » dessinait une image du milieu et du système des soins aux personnes atteintes de cancer ainsi que des changements qui avaient marqué la pratique infirmière et les populations de patients durant la décennie précédente. Le deuxième thème intitulé "Des intérêts contradictoires" se rapporte aux défis que les composantes $d u$ changement et les forces sociales ont présentés pour la pratique professionnelle des soins infirmiers en oncologie. Quant au troisième thème, "Trouver le moyen ", il décrit les types de comportement utilisés par les infirmières en oncologie pour gérer l'évolution des soins de santé et pour trouver un sens dans leur travail. Dans l'ensemble, le portrait des infirmières en oncologie canadiennes ébauché à l'aide de nos résultats, révèle qu'elles fonctionnent en mode survie. Elles font face à de nombreux défis dans leur lieu de travail et sont capables de continuer pour l'instant parce qu'elles trouvent jour après jour le moyen d'équilibrer leurs responsabilités et parce qu'elles croient et savent que leurs compétences et connaissances infirmières spécialisées leur permettent de faire la différence sur le plan des soins aux patients.

Au cours de la dernière décennie, la réforme et la restructuration des soins de santé ont entraîné des changements importants dans le milieu de travail des professionnels de la santé. Les soins en oncologie sont dispensés dans divers contextes cliniques tels que les unités de soins hospitaliers, les cliniques de soins ambulatoires et les services de soins à domicile. Dans chacun de ces contextes, la nature du travail subit, depuis les années 90 , l'influence de plusieurs facteurs incluant la mise en œuvre de changements organisationnels en vue de gérer l'amenuisement des ressources tout en devant faire face à l'accroissement de l'incidence et de la prévalence des patients atteints du cancer au sein du système. En outre, le développement des connaissances scientifiques et les progrès technologiques ont augmenté la complexité des modalités de traitement du cancer dispensées dans les contextes de soins hospitaliers ou autres.

Il ne fait aucun doute que les changements touchant le système des soins de santé et le système de soins aux personnes atteintes du cancer ont eu une incidence sur les infirmières en oncologie et sur leurs expériences professionnelles. Par contre, on n'a pas une idée nette de la manière dont les infirmières en oncologie canadienne perçoivent ces changements et de l'impact de ces derniers sur leurs milieux de travail et sur la pratique clinique. Une étude qualitative des infirmières en oncologie canadiennes a donc été effectuée dans le but d'explorer la condition d'infirmière en oncologie au cours de la dernière décennie et d'examiner la manière dont les infirmières en oncologie s'adaptent dans un système de soins en pleine évolution. Si l'on veut déterminer des interventions de soutien au personnel infirmier, on se doit d'acquérir une compréhension approfondie des changements et des défis auxquels les infirmières en oncologie font face dans leur pratique quotidienne et de recueillir des informations additionnelles sur la façon dont elles s'adaptent aux réalités du système actuel.

\section{Recension des écrits}

\section{Le vécu des infirmières en oncologie}

Au début des années 90 , les premières recherches sur l'essence des soins infirmiers en oncologie ont été lancées, aux Etats-Unis, par Cohen et ses collègues dans le cadre du Life Cycle Task Force de l'Oncology Nursing Society (Cohen, 1995; Cohen, Haberman, Steeves et Deatrick, 1994; Cohen et Sarter, 1992; Haberman, Germino, Maliski, Stafford-Fox et Rice, 1994; Steeves, Cohen et Wise, 1994). Trente-huit infirmières en cancérologie d'un peu partout aux États-Unis ont participé à une enquête ayant pour but de décrire la signification des soins infirmiers en oncologie, la manière dont les infirmières trouvent un sens à leur travail et enfin, leurs perceptions relativement aux gratifications et aux difficultés qui jalonnent le travail d'infirmière en oncologie. Les répondantes ont souligné les défis associés à la prise en charge des personnes ayant des problématiques complexes, à l'évaluation de priorités multiples et à la gestion des crises psychologiques et/ou physiologiques fréquentes et imprévues. Elles utilisent des métaphores de guerre pour décrire la «lutte » contre les effets du cancer tels que la mutilation, la souffrance et la mort. Parmi les facteurs qui donnaient une signification particulière aux soins infirmiers en oncologie, on relevait les reconnaissances

Debra Bakker, inf., PhD, Professeure, École des sciences infirmières, Université Laurentienne.

Margaret Fitch, inf., PhD, Chef, Soins infirmiers en oncologie et Soins de soutien, Centre régional de cancérologie de TorontoSunnybrook.

Esther Green, inf., M.Sc.inf., Chef de direction, Soins infirmiers et Directrice, Planification des ressources humaines en santé, Action Cancer Ontario.

Lorna Butler, inf., PhD, Professeure, École des sciences infirmières, Université Dalhousie.

Karin Olson, inf., PhD, Professeure agrégée, Faculté des sciences infirmières, Université de l'Alberta. 
professionnelles et personnelles qui accompagnaient la maîtrise des soins aux patients atteints d'une maladie complexe et l'établissement de relations d'aide et de confiance qui permettaient aux infirmières « d'être là » pour fournir réconfort et soutien aux patients et à leur entourage. Les résultats de ces études qualitatives indiquent que les soins infirmiers en oncologie sont d'une nature à la fois enrichissante et exigeante sur le plan émotif. La perception que les infirmières en oncologie avaient de la signification de leur travail et la compréhension de leur rôle semblaient être façonnées par leurs expériences personnelles et familiales du cancer, par leurs expériences professionnelles telles qu'être des modèles influents et de vivre les réalités de l'accompagnement des patients atteints du cancer alors qu'ils connaissent chagrins et triomphes (Haberman et al., 1994). L'information tirée des études qui explorent le travail des infirmières en cancérologie, le sens que ces dernières donnent à leur travail et l'utilité qu'elles perçoivent dans leur rôle professionnel, tout cela aide à mieux cerner les soins infirmiers en oncologie en tant que sous-culture ainsi que les qualités, les comportements et les compétences originaux exigés par la dispensation de soins aux patients atteints du cancer (Gambles, Wilkinson et Dissanayake, 2003; Haberman et al., 1994; Steen, Burghen, Hinds, Srivastava et Tong, 2003). C'est en développant leur connaissance de la nature du travail des infirmières œuvrant auprès des patients atteints du cancer et en découvrant l'analyse qu'elles font de leur travail et la réflexion à laquelle elles se livrent, que les infirmières gestionnaires et les infirmières enseignantes réussiront le mieux à appuyer, chez les infirmières, le développement et le rehaussement de la pratique de leur spécialité et à les soutenir dans leur travail (Corner, 2002).

\section{Agents stressants éprouvés par les infirmières en oncologie}

Les écrits définissent les infirmières en oncologie comme un groupe de professionnels de la santé qui subissent un important stress professionnel du fait de la nature de leur travail quotidien (Escot, Artero, Gandubert, Boulenger et Ritchie, 2001; Hinds et al., 1990; Larson, 1992; Lewis, 1999; Medland, Howard-Ruben et Whitaker, 2004; Wilkinson, 1994). Les travaux de recherche examinant le stress lié à l'emploi dans le domaine des soins infirmiers en oncologie se concentraient, pour la plupart, sur la détermination et la mesure de déterminants particuliers du stress (Bond, 1994; Emery, 1993; Escot et al., 2001; Florio, Donnelly et Zevon, 1998; Hinds et al., 1990; Hinds, Quargnenti, Hickey et Mangum, 1994; Kushnir, Rabin et Azulai, 1997).

Les travaux les plus systématiques ont été menés par Hinds et ses collègues (Hinds, 2000; Hinds et al., 1990; 1994) dans leur étude de la séquence stress-réaction chez les infirmières en oncologie pédiatrique. Leurs conclusions indiquaient que les infirmières en oncologie pédiatrique éprouvaient un stress lié au rôle allant de modéré à élevé et que l'intensité du stress variait en fonction du nombre d'années d'expérience dans la spécialité de l'oncologie pédiatrique et du type de milieu de pratique. Les principales sources de stress dégagées étaient les suivantes : assister au décès de patients, voir souffrir des patients et leurs proches, travailler aux côtés de collègues qui ne semblaient pas faire preuve d'un engagement ou d'un souci adéquat à l'égard des soins aux patients et enfin, exercer dans des contextes où les contributions des infirmières en oncologie pédiatriques n'étaient pas reconnues ou valorisées. En étudiant plus à fond les réactions des infirmières en pédiatrie au stress lié à leur emploi, Hinds et ses collègues (Hinds, 2000; Steen et al., 2003) ont découvert que les infirmières demeuraient dans ces « rôles à stress élevé » si elles donnaient un sens à leur travail.

Des études antérieures (McElroy, 1982; Stewart, Meyerowitz, Jackson, Yarkin et Harvey, 1982; Wilkinson, 1994) qui examinaient le stress chez des infirmières soignant des patients en oncologie adultes ont dégagé des sources de stress semblables à celles éprouvées par les infirmières en oncologie pédiatrique ainsi que d'autres agents stressants professionnels. Ces autres sources de stress comprenaient des conflits avec des membres du personnel, de l'administration et des médecins, le sentiment d'être mal préparées pour faire face aux exigences émotionnelles des patients et de leurs proches, des questions d'éthique liées aux soins aux patients et à la recherche, la surcharge de travail et la conciliation travail-vie de famille (McElroy, 1982; Wilkinson, 1994). Bien que ces sources de stress ne soient pas l'apanage des prestataires des soins en cancérologie, il a été suggéré que ces facteurs de stress surviennent plus fréquemment en oncologie que dans les autres contextes de soins parce que le travail des infirmières en cancérologie comporte une large part liée à l'aspect émotionnel (Corner, 2002; Stewart, Meyerowitz, Jackson, Yarkin et Harvey, 1982).

L'importance du nombre de facteurs de stress quotidien suggère que les infirmières en oncologie sont vulnérables à « l'épuisement professionnel ». Par épuisement professionnel, on entend un syndrome lié à l'emploi qui résulte d'une exposition prolongée à de hauts niveaux de stress et qui se caractérise par une grande fatigue, une dépersonnalisation et un faible sentiment d'accomplissement personnel (Maslach, 1976; Maslach, Schaufeli et Leiter, 2001; Penson, Dignan, Canellos, Picard et Lynch, 2000). Il survient dans des milieux où les exigences de l'emploi dépassent le soutien et les ressources dont disposent les travailleurs (Maslach et al., 2001). Cependant, les études portant sur l'épuisement professionnel chez les infirmières soignant des adultes atteints du cancer n'ont pas révélé de différences statistiquement significatives dans le niveau de stress ou le degré d'épuisement professionnel chez les infirmières en oncologie d'une part et chez les infirmières œuvrant dans d'autres spécialités, d'autre part (Jenkins et Ostchega, 1986; Papadatou, Anagnostopoulos et Monos, 1994; Yasko, 1983). Étant donné que toutes ces études ont déjà plus de dix ans, il est possible qu'elles aient été menées dans des milieux où l'impact de la réforme des soins de santé n'était pas du tout un enjeu. De même, les conclusions peuvent être le reflet de la méthode qui consiste à mesurer le stress à un seul moment. Des recherches plus récentes sur l'épuisement professionnel indiquent que les caractéristiques personnelles et l'exposition journalière aux souffrances des patients contribuent à son développement mais qu'il est tout aussi important d'examiner l'environnement social et les facteurs organisationnels dans le lieu de travail (Corner, 2002; Garret et McDaniel, 2001; Maslach et Leiter; 1998). Il est nécessaire de conduire d'autres études du stress et de l'épuisement professionnel chez les infirmières en oncologie œuvrant dans le milieu des soins de santé contemporain en constante évolution puisque les conséquences de l'épuisement professionnel peuvent avoir un effet négatif sur les prestataires de soins ainsi que sur les personnes visées par ces soins (Corner, 2002; Grunfeld, Whelan, Zitzelsberger, Willan, Montesanto et Evans, 2000; Grunfeld, Zitzelsberger, Coristine, Whelan, Aspelund et Evans, 2005; Leiter, Harvie et Frizzell, 1998).

\section{Études sur le milieu de travail infirmier}

Ces dernières années, des études publiées sur le milieu de travail infirmier décrivent l'impact de la restructuration des soins de santé sur la main-d'œuvre infirmière du Canada et d'autres pays occidentaux (Aiken et al., 2001; Aiken, Clarke, Sloane, Sochalski et Silber, 2002; Armstrong-Stassen, 2003; Armstrong-Stassen, Cameron, Horburgh, 1996; Baumann, Giovannetti, O’Brien-Pallas, Mallette, Deber, Blythe, Hibberd et DiCenso, 2001; Blythe, Baumann et Giovannetti, 2001; Burke et Greenglass, 2000; Cummings et Estabrooks, 2003; Laschinger, Sabiston, Finegan et Shamian, 2001; Laschinger, Shamian et Thomson, 2001; Maurier et Northcott, 2000). Ces études signalent que les infirmières perçoivent une perte de contrôle sur leur vie personnelle, sur leurs emplois et 
leurs possibilités de carrière et que ces changements peuvent compromettre, en bout de ligne, leur capacité de prodiguer des soins efficaces aux patients. Jusqu'à présent, les recherches sur le milieu de travail infirmier ont surtout porté sur les infirmières en tant que grand groupe professionnel et peu de travaux concernent le domaine des soins infirmiers en oncologie. Certes, l'Oncology Nursing Society a lancé, en 2000, une évaluation sur la situation de la maind'œuvre infirmière en oncologie aux États-Unis (Buerhaus, Konelan, DesRoches, Lamkin et Mallory, 2001; Lamkin, Rosiak, Buerhaus, Mallory et Williams, 2002a, b). Une enquête a été effectuée auprès d'infirmières en oncologie, d'oncologues et de chef de direction en soins infirmiers œuvrant dans des établissements de cancérologie en vue de découvrir leurs perspectives sur les modèles actuels de dotation en personnel infirmier dans les milieux de soins oncologiques aux É.-U., l'évolution des milieux de travail et l'impact des pénuries de personnel sur la qualité des soins aux patients. En revanche, aucune étude n'a été menée au Canada sur le milieu de travail infirmier, spécifiquement lié à la spécialité des soins infirmiers en oncologie.

Il existe plusieurs raisons impérieuses d'appuyer des recherches dans ce domaine, comme la présente étude qui explore la vie au travail des infirmières en oncologie du Canada. Premièrement, la réorganisation massive des systèmes de soins qui a démarré au milieu des années 90 a donné naissance à des milieux de pratique incertains pour l'ensemble des infirmières (Aiken et al., 2001, 2002; Armstrong-Stassen, 2003; Baumann et al., 2001; Blythe et al., 2001; Burke et Greenglass, 2000; Cummings et Estabrooks, 2003; Laschinger et al., 2001; Maurier et Northcott, 2000). Deuxièmement, la pénurie généralisée de personnel infirmier et la hausse soutenue du nombre de personnes atteintes de cancer, indiquée par les statistiques en cancérologie constituent de véritables menaces au maintien à long terme d'un effectif infirmier de qualité en oncologie capable de participer à la lutte contre le cancer dans notre pays. En 2002, un rapport du Comité consultatif canadien sur les soins infirmiers recommandait qu'une des grandes priorités pour remédier à la pénurie de personnel infirmier serait d'améliorer la vie au travail des infirmières. Ainsi, c'est à titre de point de départ dans la résolution des enjeux de vie au travail des infirmières en oncologie du Canada qu'une étude qualitative a été réalisée afin de cerner l'évolution des soins infirmiers en oncologie à la suite de la mise en chantier de la restructuration des soins de santé au milieu des années 90. En étudiant la manière dont les infirmières en oncologie donnent une signification à leur travail et se comportent dans le contexte culturel de la dispensation des soins aux patients atteints du cancer dans un milieu de soins à la fois changeant et incertain, on comprendra mieux l'incidence que la restructuration organisationnelle et les tendances démographiques ont eue sur cette spécialité infirmière au Canada. Les résultats de l'étude fourniront une précieuse information aux gestionnaires, administrateurs et décideurs en soins de santé qui pourront l'utiliser pour éclairer le développement des interventions ou des ressources dont les infirmières en oncologie doivent bénéficier en vue de maintenir et de promouvoir la sécurité et l'efficacité des soins aux patients et d'assurer la durabilité d'un effectif de qualité en soins infirmiers en oncologie.

\section{Méthodologie}

\section{Méthodologie de recherche}

Nous avons utilisé une approche ethnographique ciblée (Fetterman, 1998; Morse et Field, 1995; Roper et Shapira, 2000; Spradley, 1979) pour explorer la signification et l'expérience du travail d'infirmière en oncologie dans un système de soins en évolution. Utilisées comme méthodologie d'enquête, des descriptions ethnographiques ciblées de sous-groupes d'infirmières permettent «d'étudier la pratique des soins infirmiers en tant que phénomène culturel »(Roper et Shapira, 2000, p. 9). Les soins infirmiers en oncologie constituent une sous-culture qui se manifeste dans la façon dont les infirmières expriment un corpus de connaissances et de compétences cliniques acquises, partagent un vécu professionnel lié à la prestation des soins aux patients atteints du cancer et à leurs proches, comprennent les normes qui régissent leur pratique telles que les normes professionnelles et les mandats et participent à des activités reliées au pouvoir et à des relations interpersonnelles. Nous avons retenu l'ethnographie pour cette étude afin d'apprendre auprès des infirmières en oncologie la manière dont leur compréhension culturelle de leur spécialité infirmière (c.-à-d., connaissances, croyances, idées, valeurs, coutumes) éclaire leurs comportements (c.-à-d. gérer leur tâches infirmières et leurs interactions avec d'autres professionnels de la santé et les patients atteints du cancer et leur famille) alors que le système de soins traverse une période transitoire caractérisée par l'incertitude.

\section{Échantillonnage}

Nous avons fait appel à l'échantillonnage intentionnel pour trouver des infirmières en oncologie par le biais des réseaux cliniques des membres de l'équipe de recherche qui travaillaient dans différentes régions du Canada (Colombie-Britannique, Alberta, Ontario et Nouvelle-Écosse). Nous recherchions pour l'étude des infirmières autorisées qui dispensaient des soins directs à des patients atteints du cancer et avaient au moins quatre années d'expérience en soins infirmiers en oncologie. Le critère du minimum de quatre années avait été retenu pour s'assurer que les participants à l'étude avaient assimilé, grâce à leur expérience en soins infirmiers, les connaissances, valeurs et compétences particulières à la sous-culture que constitue cette spécialité infirmière (Benner, 1984). Il importait également que les participantes possèdent plusieurs années d'expérience professionnelle dans des systèmes provinciaux de soins en cancérologie qui subissaient une transformation. Au départ, chaque membre de l'équipe de recherche a recruté sept infirmières en oncologie œuvrant dans les centres de cancérologie de sa province. On a ajouté d'autres participantes à mesure que l'étude avançait afin d'obtenir une description dense des soins infirmiers en oncologie à l'échelle du Canada. Il en découle que pour satisfaire aux critères d'échantillonnage de pertinence et d'adéquation (Morse et Richards, 2002), nous recherchions activement des participants œuvrant dans des milieux naturalistes où les infirmières fournissent des soins aux patients atteints du cancer (cliniques externes, unités d'hospitalisation, services de santé communautaire/de soins à domicile) et nous avons fait en sorte qu'il y ait des infirmières « senior » et « junior »en terme d'expérience en oncologie de manière à obtenir et à comparer les perspectives représentant divers milieux cliniques, groupes d'âge et niveau d'expérience. Les participants éventuels étaient contactés individuellement par un des membres de l'équipe de recherche qui leur fournissaient des renseignements sur l'étude et répondait aux questions qu'ils avaient sur leur participation. Après avoir reçu leur déclaration de consentement éclairé, l'équipe de recherche faisait parvenir les coordonnées des participants à deux intervieweuses formées à cet effet qui prenaient alors rendez-vous avec chaque participant pour une entrevue téléphonique à un moment qui lui convenait.

\section{Collecte de données}

Étant donné que les participants vivaient et travaillaient dans des milieux de soins en cancérologie d'un peu partout au Canada, les données ont été recueillies dans le cadre d'entrevues téléphoniques. Nous avons conçu pour les entrevues un format relativement peu structuré afin de permettre aux participants de fournir des descriptions de leur vie au travail et de leur pratique professionnelle. Les questions posées lors de l'entrevue ont été élaborées par l'équipe 
de recherche et découlaient de discussions informelles, vers la fin des années 90, de la réforme des soins de santé au Canada et des stratégies de lutte contre le cancer. Avant la tenue de l'entrevue, les participants recevaient par la poste la liste des questions qu'ils pourraient approfondir dans leurs réflexions sur les expériences vécues à titre d'infirmières en oncologie. Ces questions formaient le fil directeur de l'entrevue téléphonique. Au tout début de celle-ci, on posait à chaque participant la question générale suivante : «Qu'estce que cela signifie d'être une infirmière en oncologie aujourd'hui, et en quoi l'expérience diffère-t-elle de ce qu'elle était il y a 5 à 10 ans? » Par la suite, les intervieweuses posaient des questions en vue d'inciter les participants à fournir des descriptions plus précises d'événements, de comportements, de relations et/ou d'autres interactions. Par exemple, on invitait les participants à décrire ce qui revêtait la plus grande importance dans leur pratique des soins infirmiers ou ce qui lui donnait la plus grande partie de son sens, les frustrations et les pressions qu'elles rencontraient dans leur pratique quotidienne et enfin, l'impact qu'avaient les changements apportés au système sur leur vie personnelle et professionnelle. Des données démographiques (âge, nombre d'années en soins infirmiers en oncologie, éducation et type de milieu de pratique dans lequel le participant occupait son emploi) ont été recueillies afin de décrire l'échantillon. Tous les participants ont participé à une entrevue et la durée de cette dernière variait de 30 à 60 minutes. Chaque entrevue était enregistrée sur cassette audio, et son verbatim a été transcrit par la suite.

Durant la phase de collecte des données, tous les membres de l'équipe de recherche occupaient des postes en sciences ou en soins infirmiers qui leur permettaient de faire «partie du monde des

Tableau 1. Caractéristiques des participantes à l'étude $(\mathbf{N}=51)$

\begin{tabular}{|l|l|l|}
\hline \multicolumn{2}{|l|}{ Tableau 1. Caractéristiques des participantes à l'étude (N=51) } \\
\hline Caractéristique & Fréquence & Pourcentage (\%) \\
\hline Âge (années) & & \\
\hline$\leq 30$ ans & 1 & 2,0 \\
\hline $31-40$ ans & 11 & 21,6 \\
\hline $41-50$ ans & 22 & 43,1 \\
\hline$\geq 51$ ans & 17 & 33,3 \\
\hline Éducation & & \\
\hline Diplôme d'infirmière & 34 & 66,6 \\
\hline B.Sc.inf. & 17 & 33,3 \\
\hline Maîtrise & 3 & 5,9 \\
\hline Certification en oncologie & 19 & 37,2 \\
\hline $\begin{array}{l}\text { Nombre d'années en oncologie } \\
\text { (N=49) }\end{array}$ & & \\
\hline$\leq 10$ ans & 12 & 24,5 \\
\hline $10-15$ ans & 22 & 44,9 \\
\hline $16-20$ ans & 9 & 18,4 \\
\hline $21-25$ ans & 5 & 10,2 \\
\hline$\geq 25$ ans & 1 & 2,0 \\
\hline Milieu clinique & 39 & 76,5 \\
\hline Soins ambulatoires & 9,9 \\
\hline Clinique de consultation interne & 9 & \\
\hline Milieu communautaire & 3 & \\
\hline & & \\
\hline
\end{tabular}

soins infirmiers en oncologie ». Les chercheuses avaient librement accès, dans le cadre de leurs propres responsabilités de travail, à des milieux de travail relatifs aux soins infirmiers en oncologie où elles avaient de fréquentes interactions (quotidiennes, dans le cas de certaines) avec des infirmières en oncologie, participaient au travail d'infirmières en oncologie ( $p$. ex. réunions de l'équipe soignante, réunions d'étude de cas, formation en milieu de travail) et les observaient au travail (p. ex. interactions avec des patients/proches ou avec d'autres prestataires de soins). Ainsi, quoiqu'il n'y ait pas eu d'observation planifiée et formelle des participants, les observations effectuées par les chercheuses et leur expérience du milieu des soins infirmiers en oncologie les ont aidées à mieux comprendre les événements journaliers tenus pour acquis ainsi que les pratiques et les croyances des infirmières en oncologie. Ces aperçus très pointus ont été intégrés aux données lorsque l'équipe a effectué l'analyse et l'interprétation des entrevues des participants.

\section{Analyse des données}

On a fait appel à la statistique descriptive pour élaborer un profil sommaire du groupe de participants. Les résultats qualitatifs sont basés sur une analyse thématique (Coffey et Atkinson, 1996; Fetterman, 1998; Morse et Field, 1995; Luborsky, 1994) qui privilégiait la compréhension de l'expérience des participants du milieu des soins infirmiers en oncologie - et de la signification qu'ils lui donnaient - dans le contexte des soins de santé en constante évolution. Les sujets, les habitudes et les thèmes ont été dégagés dans les versions transcrites en vue de décrire les façons dont les infirmières en oncologie exercent leur profession, les manières dont elles s'adaptent aux changements affectant leurs milieux de travail et enfin, les croyances qu'elles entretiennent à leur sujet.

Lors de la première phase de l'analyse des données, deux des chercheuses ont examiné cinq entrevues et ont annoté dans les marges les sujets émergeant des données. Ces sujets ont ensuite servi à formuler les unités de signification. Les autres membres de l'équipe ont alors examiné les mêmes entrevues afin de donner leur avis sur les sujets dégagés et de contribuer à l'élaboration d'un système de codage global pour l'analyse des verbatims restants. Deux membres de l'équipe de recherche ont poursuivi l'examen des 51 verbatims d'entrevue. Ces deux personnes ont examiné et codé, chacune de leur côté, les versions transcrites avant de se réunir et de comparer leurs travaux de codage. À l'aide d'un procédé systématique d'étude des verbatims ligne par ligne, les passages textuels ont été codés et les verbatims ont été comparés dans le but d'y déceler les habitudes des participants sur le plan des croyances, réactions et comportements. En outre, tandis que ces deux chercheuses codaient, analysaient et interprétaient les données des entrevues, les discussions périodiques tenues avec le reste de l'équipe et le partage d'observations et d'expériences relatives aux milieux de travail de l'oncologie ont favorisé de nouveaux recoupements et un nouveau classement des habitudes mises au jour. De cette façon, les perspectives de l'étique et de l'émique ont permis de faire ressortir les habitudes et les thèmes liés à la condition d'infirmière en oncologie dans un système de santé en évolution.

\section{Résultats}

\section{Caractéristiques de l'échantillon}

En tout, 51 infirmières en oncologie d'un peu partout au Canada ont participé à l'étude. Les caractéristiques démographiques de l'échantillon sont présentées dans le tableau 1. Les participants avaient entre 24 et 60 ans et l'âge moyen des membres du groupe était de 45,9 ans. Presque les trois quarts (73\%) des infirmières avaient suivi une formation collégiale et $37 \%$ des infirmières avaient obtenu la certification spécialisée en soins infirmiers en oncologie. 
Parmi les participants, le nombre d'années d'expérience en soins infirmiers en oncologie s'étendait de 4 à 26 années, la durée moyenne s'élevant à 13,8 années. Bien que l'échantillon d'infirmières en oncologie comprennent des infirmières représentant des milieux dispensant des soins en clinique interne et en clinique externe (cliniques ambulatoires et soins communautaires/à domicile), les infirmières œuvrant dans les cliniques de soins ambulatoires y étaient surreprésentées. Cette distribution particulière vient du fait que le recrutement de l'échantillon a démarré dans ces contextes de travail et qu'il était plus facile d'identifier les infirmières en oncologie exerçant dans les organismes provinciaux de soins en cancérologie et d'entrer en contact avec elles. Pour la majorité des infirmières interviewées, leur pratique des soins infirmiers concernait exclusivement la prestation de soins directs aux patients atteints du cancer. Environ $10 \%$ des membres de l'échantillon ont indiqué qu'en dehors de leurs responsabilités liées aux soins directs aux patients, leur charge de travail incluait également des activités dans les domaines de l'enseignement, du leadership et de la recherche.

\section{Thèmes}

L'analyse des données a mis en relief une image culturelle de « ce qu'est le monde (un milieu changeant et incertain) pour les individus (les infirmières en oncologie) qui ont appris à voir, à entendre, à parler et à penser de manières différentes » (Spradley, 1979, p. 3). Le premier thème, «Le milieu des soins de santé », se rapporte au contexte dans lequel œuvrent les infirmières spécialisées en oncologie du Canada. Le deuxième thème, «Des intérêts contradictoires », aborde l'impact des éléments du milieu des soins de santé sur les infirmières en oncologie; il trouve son origine dans les autoréflexions des participants et les significations qu'ils donnaient aux événements et aux interactions sociales dans l'environnement des infirmières en oncologie. Le troisième thème, « Trouver le moyen », reflète les habitudes comportementales, les croyances et les valeurs des infirmières en oncologie ainsi que le sens qu'elles donnaient à la modification de leur environnement et à leurs responsabilités professionnelles, y réagissaient et les géraient.

\section{Le milieu des soins de santé}

Lorsqu'ils décrivaient la façon dont leur milieu de travail avait changé durant la décennie, les participants ont cerné trois domaines particuliers : les profils de patients, la réorganisation du système et la pratique professionnelle des soins infirmiers.

\section{Profils de patients}

Les participants ont dégagé plusieurs changements relatifs aux profils des patients qui avaient une incidence directe sur le travail des infirmières. Les participants provenant des contextes des soins en clinique interne, des soins en clinique externe et des soins communautaires rapportaient un accroissement du volume de patients atteints du cancer ainsi qu'un accroissement de l'acuité des besoins du patient. Quelques infirmières estimaient que l'augmentation du nombre de patients dans leur contexte de travail atteignait les 30$50 \%$. Dans sa description de l'acuité des besoins des patients pris en charge dans les contextes de soins ambulatoires, une participante a déclaré :

«Nous prodiguons des soins communautaires aux patients sur une bien plus longue durée, tout simplement parce que nous n'avons plus un nombre suffisant de lits d'hôpital. Dorénavant, les patients essaient de maintenir leurs habitudes de vie normales, ils sont en ambulatoire et visitent les cliniques sur une plus longue durée qu'avant. Dans le temps, ils cessaient de venir dans les cliniques... mais maintenant, nous suivons des patients jusqu'à leur décès. »
Les infirmières signalaient que les attentes des patients et de leurs proches avaient également changé. Ces derniers possèdent de meilleures connaissances qu'il y a dix ans, ont un meilleur accès à l'information qu'ils utilisent à bon escient pour devenir des consommateurs de soins plus avertis. Bien que les participants aient indiqué qu'en général les patients appréciaient les soins qui leur étaient prodigués, ils ajoutaient que les patients semblaient avoir une conscience plus aiguë des «compressions et pénuries » au sein du système de soins. Les patients exprimaient davantage leurs inquiétudes et leur " insatisfaction ou leur frustration du fait du manque de personnel et du peu de temps que celui-ci pouvait leur consacrer. »

\section{Réorganisation du système}

Les participants ont décrit les nombreux changements qui ont touché leur contexte de travail dans le cadre de la vaste restructuration du système de soins tout au long des années 90. Quelque soit leur situation géographique au Canada, tous les participants ont fait état d'une réduction des effectifs (au niveau du personnel infirmier et du personnel de soutien) ainsi que de la perte résultante d'un personnel qualifié. Partout, la pénurie de personnel infirmier et l'accroissement du nombre de patients atteints du cancer ont entraîné un nombre de cas plus élevé pour les infirmières qui avaient conservé leur poste dans ces milieux de travail.

De plus, dans certaines situations, la réduction du personnel de soutien a obligé les infirmières à assumer des tâches additionnelles. Tous les participants ont rapporté qu'ils avaient désormais davantage de responsabilités qui ne se rapportaient pas directement aux soins au patient ni à la pratique infirmière. Une participante a ainsi déclaré :

"Je pense que nous devons effectuer bien des tâches jadis rattachées à des emplois qui ne sont plus... ils n'existent plus à cause des compressions gouvernementales. Je crois que les soins infirmiers ont de tout temps été une profession fourre-tout où les infirmières finissent par faire tout ce qui ne peut pas être fait par quelqu'un d'autre comme lorsqu'il faut emmener le patient quelque part, ou faire ceci, remplir cela, ou renouveler le stock de cette fourniture, ou aller chercher cela. Il y a une foule de tâches invisibles qui sont effectuées par les infirmières, et je crois que les gens ne sont pas au courant de ces choses invisibles et intangibles. Lorsque la personne qui accomplissait ces tâches n'est plus là, c'est à l'infirmière qu'elles échoient, sinon personne ne les fait. »

Cependant, aucun participant n'a indiqué avoir refusé d'accomplir des tâches qui ne relevaient pas normalement de l'infirmière. Les répondants commentaient que les infirmières acceptaient ces responsabilités additionnelles afin « d'assurer le bon fonctionnement des systèmes » et de veiller à ce que les patients reçoivent tous les services requis.

Dans les milieux de soins de santé communautaire, les changements apportés au système tels que la fermeture de lits dans les hôpitaux et le licenciement d'infirmières se sont traduits par un nombre plus élevé de cas et une nette augmentation de la complexité des exigences de soins. Pourtant, malgré l'accroissement du nombre de patients soignés à domicile, il n'y a eu aucune différence perceptible relativement aux fonds et aux ressources offerts pour dispenser les soins nécessaires à ces patients et à leur famille. Les infirmières décrivaient les difficultés qu'elles rencontraient dans leurs efforts de mobilisation du système et de coordination des services pour le compte de leurs patients et de leur entourage. Il semblait que les infirmières en santé communautaire disposaient de peu de soutien lorsque leurs patients avaient besoin de services ou de consultation à l'extérieur. Elles faisaient plus particulierement état du manque fréquent de communication entre les prestataires de soins de santé employés 
par différentes composantes du système de soins mais qui se partageaient la prise en charge des mêmes patients. Les participants à l'étude percevaient la fragmentation et la fragilité des soins aux patients et des liens entre les services des hôpitaux/établissements et les services de santé communautaire.

Dans la plupart des établissements de soins, les modèles de gestion ont été restructurés afin de privilégier les programmes qui appuyaient le travail d'équipes interdisciplinaires de professionnels de la santé plutôt que les programmes mettant l'accent sur la séparation des disciplines et des pratiques professionnelles. Quelques infirmières ont parlé de la disparition, au sein des organigrammes, des services de soins infirmiers et de l'intégration des infirmières dans les équipes de programmes. Elles avançaient que le leadership infirmier n'avait aucune visibilité et qu'il semblait n'y avoir aucune « directive officielle quant à la personne à qui [les infirmières devraient] adresser leurs préoccupations professionnelles ». Étant données les nouvelles responsabilités des anciennes infirmières cadres qui dépassaient le champ des soins infirmiers, certaines infirmières de chevet se sentaient abandonnées et mal préparées pour leurs nouveaux rôles où la prise de décisions joue un rôle primordial. De même, de nombreuses infirmières étaient désillusionnées quant au peu de valeur que les cadres supérieurs accordaient aux questions relatives à la vie au travail du personnel infirmier. Les participants ont indiqué que c'est auprès d'autres infirmières de chevet qu'elles recherchaient de l'aide pour les enjeux relatifs aux soins aux patients et au milieu de travail. Mais leur charge de travail limitait sérieusement le temps qu'elles pouvaient consacrer à cette entraide collégiale. Beaucoup d'infirmières de chevet ont signalé l'arrêt des réunions du personnel régulières au cours desquelles on discutait des inquiétudes et des préoccupations. D'ailleurs, la perception d'un grand nombre de participants était que les « soins infirmiers ne figuraient pas parmi les points importants du programme de leur établissement en ce qui a trait au domaine de l'oncologie ».

Par contre, d'autres infirmières affirmaient que l'adoption d'un modèle de gestion axé sur les programmes au détriment d'un modèle axé sur les disciplines constituait un changement positif. Elles jugeaient que ce changement encourageait les infirmières à établir des réseaux et à négocier avec les autres prestataires de soins de santé du système. Ces infirmières ont déclaré qu'elles ne voulaient pas que quelqu'un d'autre soit là pour résoudre leurs problèmes indépendamment d'elles ni pour assumer toute la responsabilité, mais qu'elles désiraient davantage d'autonomie et d'encadrement sur le plan de la prise de décisions, particulièrement celles concernant les soins aux patients. Elles estimaient que le système habilitait les infirmières à devenir des membres de l'équipe soignante aux côtés des représentants d'autres disciplines dans la planification des programmes et des modèles pour la prestation des soins en cancérologie. Le rôle de gestionnaire en tant que facilitatrice est illustré dans le commentaire suivant :

"Les infirmières gestionnaires transforment la vision qu'elles ont d'elles-mêmes. Je pense qu'elles perçoivent désormais leur rôle comme étant celui de facilitatrices, de guides et de mentors. Elles considèrent de plus en plus les infirmières de chevet à titre d'experts cliniques. Elles prêtent davantage attention aux questions opérationnelles des infirmières de chevet... elles consultent beaucoup plus les personnes qui prodiguent directement les soins. »

\section{Pratique professionnelle des soins infirmiers}

La perception globale était que l'exercice des soins infirmiers en oncologie avait changé de manière significative au cours de la décennie. Bon nombre des changements étaient perçus positivement puisqu'ils permettaient aux infirmières en oncologie d'exercer avec une autonomie accrue au sein de l'équipe de lutte contre le cancer. Plusieurs infirmières ont parlé d'une transition en soins infirmiers où les infirmières sont passées de l'état de «travailleuses » à celui de
« professionnelles » et où l'on encourage les infirmières à " manifester leur esprit critique et à mettre fin à bien des traditions associées à l'accomplissement des tâches infirmières ».

Par contre, quelques infirmières trouvaient qu'il n'y avait eu aucun changement dans la perception de leur propre rôle par les autres professionnels du système de soins. Elles regrettaient que les membres d'autres disciplines ne valorisaient pas le savoir infirmier, qu'ils ne comprenaient pas l'interaction entre l'infirmière et le patient et qu'ils croyaient que le rôle de l'infirmière consistait à faciliter le travail d'autres professionnels de la santé.

"L'étendue de ma pratique et ce qui est attendu de moi manquent encore de clarté... les médecins ne comprennent vraiment pas ce que sont les soins infirmiers... Ils ne parviennent pas à comprendre pourquoi on peut avoir besoin d'une infirmière [dans un contexte particulier]. Ce n'est pas pour leur propre bénéfice car, en réalité, ce sont les patients qui ont besoin d'une infirmière. Ils ne comprennent pas la nature de la relation patients-infirmières...»

Tous les participants indiquaient que les attentes relatives à l'étendue de leur pratique des soins infirmiers avaient bel et bien évolué. Plusieurs facteurs avaient favorisé ce changement tels que l'adoption d'un modèle de prestation des soins en cancérologie qui sont dorénavant prodigués principalement à l'extérieur des hôpitaux de soins actifs, l'implantation des modèles de soins infirmiers intégraux ou de gestion de cas dans de nombreux contextes de soins en consultation interne et externe ainsi que les progrès scientifiques et technologiques qui ont donné naissance à de nouveaux protocoles de traitement du cancer. Les infirmières ont fait état du besoin qu'elles avaient d'acquérir des connaissances et des compétences spécialisées. Pour dispenser des soins aux cas complexes, les infirmières en oncologie devaient non seulement maîtriser des compétences et des techniques relatives aux soins physiques mais encore acquérir des connaissances additionnelles sur l'évaluation et la surveillance des patients vivant en milieu communautaire, sur l'enseignement et le counseling des patients et de leurs proches, sur le rôle de porte-parole des patients et sur la coordination des services de soutien. En outre, si elles voulaient assurer leur survie dans le milieu de travail transformé par la réforme des soins de santé, les infirmières devaient posséder des compétences en informatique, être capables de gérer d'autres travailleurs de la santé et de collaborer avec d'autres professionnels de la santé et négocier, avec eux, sur certaines questions de soins aux patients. Un critère mentionné par les infirmières a été la mise en œuvre, en 1997, de l'examen de certification en soins infirmiers en oncologie de l'Association des infirmières et infirmiers du Canada. Beaucoup de participants à l'étude ont signalé qu'ils avaient obtenu leur certification de spécialité et que leurs collègues faisaient de ce but une grande priorité. Elles croyaient fermement que l'établissement de la spécialité des soins infirmiers en oncologie avait rehaussé la situation des soins infirmiers en oncologie au sein du système.

\section{Des intérêts contradictoires}

Le deuxième thème qui s'est dégagé des données a été baptisé «Des intérêts contradictoires ». Il se rapporte à la façon dont les normes culturelles (la pratique professionnelle des soins infirmiers en oncologie) et les forces sociales (les éléments du milieu des soins de santé) étaient fréquemment incompatibles. Unanimement, les participants affirmaient que les changements apportés au système créaient souvent des conditions de conflit pour la pratique professionnelle des soins infirmiers.

\section{Exercer pleinement la pratique ou travailler dans un système restructuré de nature restrictive}

Le conflit était particulièrement manifeste lorsque les changements organisationnels étaient perçus par les infirmières comme autant de contraintes au plein exercice de leur pratique. 
Dans leurs descriptions du rôle des infirmières en oncologie, les participants énuméraient les responsabilités liées à la pratique clinique, à la recherche, à l'enseignement et au leadership, en ajoutant que les soins infirmiers en oncologie ne se limitaient pas aux seuls soins physiques dispensés aux patients atteints du cancer, mais qu'ils comprenaient également les soins psychosociaux prodigués à ces patients et à leurs proches. Les infirmières décrivaient comment le processus de restructuration avait entraîné des changements tels que « pas assez d'infirmières », « le démantèlement des services de soins infirmiers » et «l'absence d'un leadership infirmier visible », qui exerçaient une incidence directe sur la pratique professionnelle des soins infirmiers. Ces conditions étaient considérées comme des obstacles au plein exercice de la pratique professionnelle des soins infirmiers et elles influençaient la façon dont les connaissances des infirmières et leurs contributions aux soins aux patients étaient perçues par les autres groupes de prestataires de soins de santé et par conséquent, la valeur que ces derniers y accordaient.

Les infirmières décrivaient l'introduction, durant les années 90, du modèle de soins infirmiers intégraux dans les milieux des soins en cancérologie et la façon dont il promouvait manifestement le rôle de l'infirmière au sein de l'équipe soignante en tant que travailleuse du savoir. A l'opposé des autres modèles de soins infirmiers conventionnels principalement axés sur les compétences techniques et sur la délégation de tâches, le modèle de soins infirmiers intégraux, aux dires des infirmières, confirmait l'autonomie et la responsabilité des soins infirmiers auprès des autres prestataires de soins de santé. Ainsi, elles voyaient dans l'implantation des soins infirmiers intégraux dans les milieux de la cancérologie une occasion de maximiser l'étendue de la pratique et de «montrer la différence que les infirmières peuvent faire sur le plan de la qualité des soins aux soins aux patients ». Par contre, les infirmières décrivaient les frustrations et les déceptions qu'elles ont ressenties lorsque la restructuration a entraîné des changements $\mathrm{au}$ système sans pour autant fournir l'infrastructure ni les ressources nécessaires au soutien ou à la promotion du modèle de soins intégraux.

Par exemple, dans certains milieux des soins en cancérologie, les infirmières ont été démoralisées par l'abandon du modèle de soins infirmiers intégraux parce qu'on jugeait qu'il coûtait « trop cher ». Dans d'autres cas, les soins infirmiers intégraux n'ont pas été officiellement abandonnées mais, du fait des mises à pied de personnel, on exigeait des infirmières qu'elles assument des tâches accessoires, ce qui les empêchaient d'utiliser leurs connaissances infirmières pour prendre en charge les patients et participer à la prise de décision clinique. Il est possible que les entraves au plein exercice de la pratique infirmière résultent de conflits de pouvoirs entre le « système » et la « profession ». Les participants exprimaient fréquemment l'opinion selon laquelle les infirmières sont liées à un système où les attentes officielles relatives au travail des infirmières n'appuient ou ne promeuvent pas nécessairement leur autonomie et leur responsabilité professionnelles.

«Il est extrêmement frustrant de voir qu'il n'est pas toujours possible pour les infirmières ouvrant dans un contexte de soins ambulatoires d'avoir un contact avec les patients comme elles devraient le faire du simple fait de la nature du travail des infirmières en milieu ambulatoire qui est devenu administratif avant tout... ces infirmières sont extrêmement bonnes et elles ont montré que si on les laisse dispenser des soins infirmiers la majorité de leur temps au travail, elles font une différence appréciable au niveau de la qualité de vie des clients. Mais chez de nombreuses infirmières, il n'y a qu'une infime partie de leur journée de travail où leur expertise est utilisée dans le cadre des soins aux patients. »
Répondre aux besoins individuels des patients ou gérer son temps

L'autre intérêt contradictoire dégagé concernait le peu de temps dont les infirmières disposaient pour dispenser des soins personnalisés aux patients. Les infirmières avançaient que pour elles, aller au travail, c'était « lutter contre des incendies » ou « réparer les choses ». Chaque jour, l'accent était mis sur la protection de l'intégrité du lieu de travail plutôt que d'établir des rapports avec les patients et d'apprendre à les connaître. On demandait aux infirmières d'établir des priorités et de prendre des décisions sur ce qui devait vraiment être accompli au cours de la journée de travail. Pour les infirmières surmenées, cela signifiait que les contraintes de temps les forçaient à choisir entre dispenser des soins intégrés ou concentrer leur attention sur les priorités de soins survenues ce jour-là.

"Les contraintes de temps nous font choisir entre dispenser des soins intégrés ou nous concentrer uniquement sur les priorités. Il nous faut réévaluer et même réduire nos attentes en matière de soins. Que pouvons-nous offrir de façon réaliste, durant le temps dont nous disposons, aux nombreux patients que nous soignons? Pouvons-nous nous donner la permission de nous dire que nous ne pourrons plus faire ceci ou cela. Et cela va être rudement difficile parce que les infirmières veulent effectuer la tâche globale, les soins intégraux, mais on ne leur donne plus le temps nécessaire pour le faire. »

\section{Trouver le moyen}

Le troisième thème, « Trouver le moyen », reflète les habitudes comportementales, les croyances et les valeurs des infirmières en oncologie au sein de la culture changeante des soins de santé. Lorsqu'elles décrivaient ce à quoi ressemble le métier d'infirmière en oncologie dans le monde contemporain, les infirmières faisaient état des façons dont elles s'adaptaient aux demandes du milieu de travail et donnaient un sens à leur travail dans le système des soins en cancérologie.

\section{Un numéro d'équilibriste}

Les participants décrivaient comment ils avaient appris à surmonter le conflit entre le rôle professionnel qu'ils souhaitaient exercer et les ressources limitées offertes par le système. Elles rapportaient le besoin d'équilibrer les pressions temporelles et l'accomplissement de leurs tâches liées aux soins aux patients et de leurs autres tâches. Les infirmières se livraient à un "numéro d'équilibriste » quotidien afin d'éprouver un sentiment de satisfaction à l'idée d'avoir répondu aux besoins de leurs patients tout en ayant assuré le bon fonctionnement des opérations.

«Il y a deux choses qui se passent en même temps dans le domaine clinique. Vous subissez une pression... la pression personnelle d'assurer le bon fonctionnement de la clinique et de voir les patients en temps opportun et puis la pression des besoins individuels des patients. On essaie donc de concilier tout cela. On tente toujours de prodiguer la même qualité de soin mais on ne peut pas y arriver. Il nous faut donc faire un numéro d'équilibriste et voir ce que l'on peut laisser de coté."

Beaucoup ont indiqué qu'il leur fallait réaliser, en leur for intérieur, qu'il ne s'agissait pas nécessairement d'un échec personnel, mais plutôt d'un problème à l'échelle du système, lorsqu'ils estimaient ne pas avoir réussi à fournir des « soins infirmiers de bonne qualité » à des patients individuels. Ils n'en avaient tout simplement pas le temps.

En outre, plusieurs infirmières ont fait état du besoin constant qu'elles avaient de maintenir un équilibre entre leur vie au travail et leur vie personnelle à la maison. Les infirmières éprouvaient souvent de la difficulté à prendre du recul à la fin de la journée par rapport à la charge de travail émotionnelle qui accompagne la prestation des soins aux patients atteints d'une maladie complexe. De plus, quelques 
participants ont ajouté que leurs situations de travail actuelles n'étaient pas saines et qu'ils avaient de la difficulté à maintenir un équilibre acceptable entre travail et vie personnelle.

\section{Faire la différence}

Malgré les frustrations associées à leur travail quotidien, les infirmières participantes ont fait part de la passion qu'elles éprouvent pour les soins infirmiers en oncologie. Elles croyaient qu'il y avait une différence entre les infirmières en oncologie et les autres infirmières. Elles attribuaient l'expression « unique en son genre » à la spécialité des soins infirmiers en oncologie et précisaient que la prise en charge des patients atteints du cancer était, pour elles, un «privilège » du fait des rapports et des liens que les infirmières nouent avec les patients et leur entourage tout au long de la maladie. Elles jugeaient que les infirmières en oncologie possédaient un plus profond sens de l'engagement envers les patients et qu'elles apprenaient, jour après jour, quelque chose de leurs interventions auprès de ces derniers. Les infirmières donnaient un sens aux défis relatifs à leur rôle et à leur milieu de travail en les comparant à l'expérience du cancer.

" Être une infirmière en oncologie est une expérience enrichissante... qui nous remplit aussi d'humilité. Nous ruminons des pensées sur les grandes pressions et frustrations qui caractérisent notre vie et nous les comparons à l'expérience que les patients doivent vivre avec leur cancer, et nous oublions vite nos petits problèmes. Il m'est arrivé tant de fois, quand je ressens de la frustration et de la colère envers ceci [ce milieu de travail]... cela m'aide beaucoup de rétablir une perspective d'ensemble de ma vie personnel et professionnelle lorsque je vois ce que les patients doivent subir.»

Tous les participants croyaient fermement que leurs compétences et connaissances spécialisées faisaient une différence dans la vie des patients atteints du cancer. Chacun des participants a partagé un récit positif illustrant la façon dont ils exercent un impact sur la qualité de vie des patients en tissant des liens avec les patients et leurs proches, en prodiguant des soins physiques et des soins de soutien et en défendant leur intérêt. On sentait que ces infirmières demeuraient dans la profession à cause des patients et qu'elles «toléraient les conditions de travail » grâce à la satisfaction personnelle qu'elles tiraient de savoir qu'elles avaient apporté une aide réelle à un individu et à sa famille.

«...c'est le fait de savoir que mon rôle d'infirmière en oncologie me permet de faire une différence au niveau des résultats pour le patient. Lorsque je leur enseigne quelque chose et qu'ils rencontrent très peu de problèmes ou que leurs symptômes sont minimisés, je sais que j'ai joué un rôle et que mon intervention les a aidés. »

La reconnaissance de la différence qu'elles faisaient sur le plan de la qualité de vie des patients et de leur vécu du cancer débouchait sur la satisfaction à l'égard de leur emploi. Pour les infirmières, les indicateurs de relations thérapeutiques efficaces étaient dégagées dans le cadre des interactions professionnelles et personnelles qu'elles entretenaient avec leurs patients et leurs proches tout au long de l'épreuve du cancer. Au beau milieu de la confusion régnant dans un système qui présente des défis journaliers sur le plan des ressources, les interactions patient-infirmière positives servaient à stimuler le moral des infirmières et à le soutenir. Ces expériences incarnaient la signification authentique des soins infirmiers en oncologie et renforçaient, chez les participants, que ce qu'elles « faisaient jour après jour en valait la peine ».

\section{Discussion}

Les descriptions fournies par les participants à l'étude indiquent que le milieu des soins infirmiers en oncologie s'est largement transformé au cours de la dernière décennie. On constatait des changements manifestes au niveau du système de soins, du profil des patients atteints de cancer et du rôle professionnel de l'infirmière en oncologie. Ces changements présentent des défis quotidiens aux infirmières en oncologie qui s'efforcent de dispenser des soins infirmiers intégraux aux patients atteints du cancer dans les contextes de soins actifs, ambulatoires et communautaires. En outre, les résultats de l'étude rendent une image des infirmières en oncologie du Canada en « mode survie ». Elles sont capables de poursuivre leurs efforts «pour le moment » parce qu'elles trouvent le moyen d'équilibrer leurs responsabilités sur une base quotidienne et qu'elles savent, en leur for intérieur, que leurs compétences et connaissances spécialisées font une différence sur le plan des soins aux patients.

\section{Valoriser le travail des infirmières en oncologie}

Au cours de la dernière décennie, de nombreuses études sur les milieux de travail des infirmières ont fait état de l'impact de la réforme des soins de santé sur la vie au travail des infirmières. Dans toutes les études publiées jusqu'à présent, les infirmières ont exprimé les mêmes préoccupations (Aiken et al., 2001; Aiken et al., 2002; Armstrong-Stassen, 2003; Armstrong-Stassen et al., 1996; Baumann et al., 2001; Blythe et al., 2001; Burke et Greenglass, 2000; Cummings et Estabrooks, 2003; Laschinger et al., 2001a,b; Maurier et Northcott, 2000). Les attentes quotidiennes liées à la prise en charge d'un nombre accru de cas par un effectif décroissant d'infirmières autorisées qualifiées auxquelles s'ajoutent des responsabilités supplémentaires relatives à des tâches non infirmières ont fait naître, chez les infirmières, un sentiment généralisé de perte de contrôle. Bien que la majorité des études aient principalement porté sur la main-d'œuvre infirmière globale, de récentes études étatsuniennes examinant la situation des soins infirmiers en oncologie (Buerhaus et al., 2001; Lamkin et al., 2002a,b) et la présente étude canadienne confirment que les infirmières qui exercent la spécialité des soins en cancérologie connaissent des attitudes et des défis similaires. Toutefois, en ce qui concerne les infirmières en oncologie du Canada, le sentiment d'impuissance et de frustration vis-à-vis du contrôle du milieu de travail a été exacerbé par des développements parallèles au niveau du rôle professionnel des soins infirmiers en oncologie. Depuis la fin des années 90, les soins infirmiers en oncologie sont reconnus au Canada comme étant une spécialité des soins infirmiers, ce qui signifie que ce rôle exige des connaissances et des compétences spécialisées. Saluée comme un événement historique, cette reconnaissance officielle a été fort bien accueillie par les infirmières en oncologie qui ont saisi l'occasion de contribuer aux efforts de l'équipe des soins en cancérologie en prodiguant des soins infirmiers intégrés. Mais, dans de nombreux contextes de soins, les changements apportés au système ont entravé le plein exercice, par les infirmières en oncologie, de leur pratique puisqu'on demandait aux infirmières professionnelles d'accomplir les tâches de nature technique ou administrative d'autres prestataires de soins de santé dont les postes avaient disparu dans le cadre des compressions.

Les multiples facettes et la nature complexe du cancer exigent des professionnels de la santé soignant les patients atteints du cancer qu'ils possèdent des connaissances et des compétences particulières en vue de répondre aux besoins de cette population de patients. L'infirmière en oncologie joue un rôle intégral dans la gestion et la coordination des soins aux patients atteints du cancer tout au long de la trajectoire de la maladie. À la lumière de la pénurie actuelle d'infirmières autorisées et de la hausse du nombre de patients atteints du cancer, il est essentiel que soit reconnu le plein potentiel du travail infirmier en oncologie. De plus, une récente étude d'Aiken et al. (2002) révèle que les niveaux de dotation en personnel infirmier ont une incidence sur les résultats pour le patient et sur le maintien des effectifs infirmiers. Des ratios patients-infirmière élevés entretenaient une corrélation importante avec la mortalité des patients et l'échec des secours (mortalité à la suite de complications), et avec de hauts niveaux d'épuisement émotionnel et une plus grande insatisfaction 
professionnelle chez les infirmières. Il est donc impérieux que les décideurs du milieu de travail consultent les infirmières en oncologie afin de structurer les contextes de travail de manière à ce que l'expertise des infirmières en oncologie soit valorisée et fasse l'objet d'une utilisation efficace au sein de l'équipe soignante dans le but de répondre aux besoins des patients atteints de cancer dont le nombre ne fait que croître.

\section{Le stress et l'épuisement professionnel}

Les soins infirmiers en oncologie sont considérés comme une profession stressante du fait de la nature des interactions avec les patients atteints d'une maladie complexe dont l'issue est fréquemment mortelle (Larson, 1992; Lewis, 1999). Les conditions de travail instables qui ont fait suite à la réforme des soins de santé ont encore aggravé la quantité de stress subie par les infirmières en oncologie du Canada. Dans la présente étude, les infirmières rapportaient que leur stress ne provenait pas de leurs interactions avec les sujets atteints de cancer mais plutôt du temps insuffisant qu'on leur donnait pour fournir le niveau de soins souhaité afin de répondre aux besoins spécifiques des patients. Cette opinion est conforme aux écrits actuels qui suggèrent que l'épuisement professionnel trouve son origine dans le fossé qui sépare les attentes des individus relativement à l'exécution de leur rôle professionnel et la structure organisationnelle existante (Leiter, 1991).

Les recherches antérieures signalent que le manque de systèmes de soutien au niveau des cadres et des compagnons de travail, le manque d'autonomie et une mauvaise communication sont les prédicteurs de l'épuisement professionnel (Albrecht, 1982; Leiter, Harvie et Frizzell, 1998; Leveck et Jones, 1996; Maslach et Leiter, 1998, 1976; Oehler, Davidson, Starr et Lee, 1991; Robinson, Roth, Keim, Levenson, Flentje et Bashor, 1991). L'analyse des verbatims des entrevues permet de mettre en évidence la présence de ces facteurs dans les milieux de travail de beaucoup d'infirmières en oncologie. Il convient donc de voir un sérieux avertissement dans les descriptions du travail infirmières en oncologie. Si l'on veut prévenir l'épuisement professionnel chez les infirmières, il est urgent d'adopter des mesures relatives au lieu de travail qui appuient le travail des infirmières en oncologie. Il a été suggéré que dans un environnement social solidaire, l'épuisement professionnel ne survient pas même si le travail est extrêmement stressant (Pines et Kanner, 1982).

\section{La culture organisationnelle}

Durant la dernière décennie, les modèles de gestion des établissements de santé ont été restructurés afin de privilégier les programmes appuyant les équipes interdisciplinaires de professionnels de la santé plutôt que des disciplines individuelles. Pour beaucoup des infirmières participant à la présente étude, cela s'est soldé par l'absence d'un leadership visible qui a débouché sur la méfiance organisationnelle. D'après Porter-O'Grady et Malloch (2003), il est nécessaire d'élaborer une nouvelle culture organisationnelle pour le siècle actuel qui mettrait l'accent sur l'atteinte des résultats voulus plutôt que sur la simple exécution des bons processus. Mais une telle transformation du cadre organisationnel peut facilement provoquer des conflits entre les travailleurs de la santé et les gestionnaires et favoriser le climat de méfiance si les chefs de file ne parviennent pas à interpréter et à communiquer les changements pour le compte d'autrui. Les réalités opérationnelles des milieux de travail contemporains obligent les infirmières à se voir et à se présenter d'une façon différente aux gestionnaires (Porter-O'Grady et Malloch, 2003). Les infirmières doivent reconnaître qu'elles sont des « travailleuses de la connaissance » et modifier la façon dont elles peuvent se faire « entendre » par les gestionnaires. Les infirmières participant à la présente étude reconnaissaient que les connaissances spécialisées qu'elles possédaient contribuaient à l'atteinte des résultats pour le patient et voulaient qu'on leur donne la possibilité d'agir en ce sens en obtenant davantage d'autonomie dans la prestation des soins aux patients ainsi qu'un contrôle sur leur environnement de pratique. Mais, dans l'ensemble, elles ne savaient pas comment être convaincantes au sein de la culture organisationnelle ou faisaient preuve d'incertitude à ce sujet. Si les gestionnaires veulent promouvoir le changement et favoriser l'adaptation des infirmières à une nouvelle culture organisationnelle, ils doivent développer leurs compétences et connaissances pour mener les autres dans un «monde fluide » en reconnaissant l'impact des changements sur la capacité des infirmières à accomplir leur travail (Porter-O'Grady et Malloch, 2003).

Il a été démontré que le soutien organisationnel est un des facteurs les plus importants en vue d'aider le personnel infirmier à faire face aux difficiles transitions de restructuration. Les conclusions de la présente étude suggèrent le besoin évident qu'il y a d'articuler les objectifs organisationnels et d'accroître la visibilité de l'appui au leadership (Burke et Greenglass, 2000). Pour favoriser le confiance organisationnelle, les gestionnaires doivent mettre l'accent moins sur le contrôle et davantage sur la coordination, l'intégration et la facilitation du travail des infirmières. Les cadres se doivent d'épouser et de parrainer les efforts visant à revitaliser les milieux de travail au sein de leurs organismes.

Malgré la transformation organisationnelle survenue dans le système de soins aux personnes atteintes du cancer au cours de la dernière décennie, les infirmières en oncologie de la présente étude sont restées fidèles à leur engagement envers les soins aux patients. Pas une seule des infirmières en oncologie interviewées n'a déclaré qu'elle projetait de quitter le domaine des soins infirmiers en oncologie. L'occasion qu'elles avaient de mettre à profit leurs connaissances et compétences spécialisées pour prendre en charge les patients atteints du cancer leur apportait un haut niveau de satisfaction. La satisfaction personnelle qu'elles tiraient individuellement de leurs interactions avec les patients atteints du cancer et leurs proches et des soins qu'elles leur prodiguaient les maintenaient à flot et leur prouvaient que leurs interventions entraînaient des résultats positifs pour le patient. D'ailleurs, il semblait, à un niveau plus fondamental, que certaines infirmières en oncologie éprouvaient de l'amour-propre à pouvoir gérer la confusion qui régnait dans leur milieu de travail tout en faisant une différence au niveau de la qualité de vie des patients. Les conclusions de cette étude soulignent l'importance de la création d'environnements où les infirmières sentent qu'elles disposent de l'autonomie nécessaire pour exercer pleinement leur pratique et où elles éprouvent des sentiments positifs et de la confiance à l'égard du travail qu'elles accomplissent.

\section{Implications pour les soins infirmiers}

Cette étude qualitative vient gonfler les rangs des écrits toujours plus nombreux sur les milieux de travail des infirmières. Les résultats indiquent que la réforme des soins de santé qui mettait l'accent sur la restructuration, la réorganisation et la réduction des effectifs a profondément touché la qualité des milieux de travail des infirmières en oncologie du Canada. Les infirmières en oncologie subissent de hauts niveaux de stress lorsqu'elles gèrent les changements et tentent de donner une signification à leur rôle d'infirmière en oncologie. L'image des infirmières en oncologie fonctionnant en «mode survie » est alarmante et elle exige qu'on s'en occupe immédiatement. Étant donné la pénurie actuelle de personnel infirmier (CNA, 2002) et l'accroissement régulier de la prévalence et de l'incidence du cancer (NCIC, 2002), il est raisonnable d'avancer qu'à l'avenir, une grande partie de la demande de services infirmiers viendra du domaine de l'oncologie. Il importe donc de prêter une attention particulière aux contextes de travail en soins infirmiers en oncologie en vue de maximaliser les compétences et la productivité des infirmières en oncologie œuvrant actuellement au sein du système de soins aux personnes atteintes du cancer et d'attirer de nouvelles infirmières vers ce domaine. 
Il a été déterminé que l'amélioration de la vie au travail des infirmières est la clé principale de la résolution de la pénurie de personnel infirmier et l'insatisfaction professionnelle généralisée qui sévit dans l'ensemble du pays (CNAC, 2002). Les résultats de cette étude fournissent quelques suggestions qui éclairent les types d'interventions qui pourraient être élaborés afin d'appuyer les infirmières en oncologie dans leur travail. Par exemple, les interventions ciblant la charge de travail des infirmières pourraient comprendre une considération du «temps » de travail. Les infirmières cadres doivent élaborer des plans autour de « qui devrait être utilisé et la manière dont chacune de ces personnes devrait être utilisée » plutôt que de chercher à obtenir davantage de ressources ou de temps (Porter-O'Grady et Malloch, 2003, p. 274).

Les résultats de cette étude et ceux d'autres travaux de recherche portant sur les prestataires de soins en cancérologie en général (Grunfeld et al., 2000; 2005) suggèrent fortement qu'il est impérieux d'instituer des stratégies de réduction ou de prévention du stress lié à l'emploi ou de l'épuisement professionnel au sein du milieu des soins aux personnes atteintes de cancer. Ces interventions devraient être conçues pour prendre en compte non seulement l'environnement de travail mais aussi la dynamique de l'équipe multidisciplinaire de soins en cancérologie (Medland et al., 2004). Ainsi, les infirmières qui forment le plus grand bloc de professionnels de la santé en cancérologie ont tout intérêt à apprendre à utiliser leur voix collective pour prendre en charge l'amélioration de la culture des soins en cancérologie. Les infirmières de tous les niveaux doivent apprendre à

\section{Références}

Aiken, L.H., Clarke, S., Sloane, D., Sochalski, J., Busse, R., Clarke, H., et al. (2001). Nurses' reports on hospital care in five countries. Health Affairs, 20(3), 43-54.

Aiken, L.H., Clarke, S.P., Sloane, D.M., Sochalski, J., \& Silber, J.H. (2002). Hospital nurse staffing and patient mortality, nurse burnout, and job dissatisfaction. Journal of the American Medical Association, 288(16), 1987-1993.

Albrecht, T. (1982). What job stress means for the staff nurses. Nursing Administration Quarterly, 7(1), 1-11.

Armstrong-Stassen, M. (2003). Dimensions of control and nurses' reactions to hospital amalgamation. The International Journal of Sociology and Social Policy, 23(8/9), 104-128.

Armstrong-Stassen, M., Cameron, S.J., \& Horburgh, M.E. (1996). The impact of organizational downsizing on the job satisfaction of nurses. Canadian Journal of Nursing Administration, 9(4), 832.

Association des infirmières et infirmiers du Canada. (2002). Planifier pour demain : Prévisions de ressources humaines en soins infirmiers. Ottawa, Ont.

Baumann, A., Giovannetti, P., O’Brien-Pallas, L., Mallette, C., Deber, R., Blythe, J., et al. (2001). Healthcare restructuring: The impact of job change. Canadian Journal of Nursing Leadership, 14(1), 14-20.

Benner, P. (1984). From novice to expert: Excellence and power in clinical nursing practice. Menlo Park, CA: Addison-Wesley.

Blythe, J., Baumann, A., \& Giovannetti, P. (2001). Nurses' experiences of restructuring in three Ontario hospitals. Journal of Nursing Scholarship, 33(1), 61-68.

Bond, D.C. (1994). The measured intensity of work-related stressors in pediatric oncology nursing. Journal of Pediatric Oncology Nursing, 11(2), 44-52.

Buerhaus, P., Konelan, K., DesRoches, C., Lamkin, L., \& Mallory, G. (2001). State of the oncology nursing workforce: Problems and implications for strengthening the future. Nursing Economics, 19(5), 198-208. faire des analyses critiques et à utiliser l'action politique pour changer la nature et la réalité du travail infirmier. Il ne suffit pas de cerner les tensions et les contradictions existantes; il faut lancer une action généralisée partant de la base en vue de reformuler les politiques et de transformer les pratiques dans le but d'améliorer les milieux de travail pour les disciplines liées à l'oncologie, ce qui se traduira par un rehaussement de la qualité des soins en cancérologie. Par action généralisée, on entend s'assurer de posséder une information complète et à jour, de bien connaître les sources de pouvoir et d'influence, de faire sentir sa présence en étendant la sphère d'influence des soins infirmiers au sein de comités interdisciplinaires, de participer à l'évaluation de politiques et de directives basés sur des données probantes et les résultats et ce, aussi bien au niveau du service qu'au niveau de l'établissement (Clarke, 2006).

Du point de vue de la recherche, les résultats de cette étude qualitative ont servi à étayer de nouvelles études par les auteures. Des travaux sont en cours pour examiner le lieu de travail plus en détail ainsi que les facteurs liée à la pratique professionnelle dans les milieux de travail des infirmières en oncologie afin de déterminer les facteurs qui exercent une influence sur le recrutement et le maintien en postes des infirmières en oncologie. Nous obtiendrons les vues des infirmières en oncologie du Canada au moyen d'enquêtes et de groupes de réflexion et elles contribueront à l'élaboration de stratégies relatives au milieu de travail qui aborderont - et éclaireront - la politique concernant la planification des ressources humaines dans les soins infirmiers en oncologie.
Burke, R.J., \& Greenglass, E.R. (2000). Effects of hospital restructuring on full-time and part-time nursing staff in Ontario. International Journal of Nursing Studies, 37(2), 163-71.

Clarke, H. (2006). Health and nursing policy: A matter of politics, power, and professionalism. In M. McIntyre, E. Thomlinson, \& C. McDonald, (Eds.), Realities of Canadian nursing: Professional, practice and power issue (2nd ed.) (pp. 72-90). Philadelphia: Lippincott Williams \& Wilkins.

Coffey, A., \& Atkinson, P. (1996). Making sense of qualitative data. Thousand Oaks, CA: Sage Publications.

Cohen, M.Z. (1995). The meaning of cancer and oncology nursing: Link to effective care. Seminars in Oncology Nursing, 11(1), 59-67.

Cohen, M.Z., Haberman, M.R., Steeves, R., \& Deatrick, J.A. (1994). Rewards and difficulties of oncology nursing. Oncology Nursing Forum, 21(8), 9-17.

Cohen, M.Z., \& Sarter, B. (1992). Love and work: Oncology nurses' view of the meaning of their work. Oncology Nursing Forum, 19(19), 1481-1486.

Comité consultatif canadien sur les soins infirmiers (CCCSI), Santé Canada, Comité consultatif sur les ressources humaines en santé. (2003, Août). Notre santé, notre avenir : un milieu de travail de qualité pour les infirmières canadiennes. Rapport final du CCCSI, www.hc-sc.gc.ca

Corner, J. (2002). Nurses' experiences of cancer. European Journal of Cancer Care, 11, 193-199.

Cummings, G., \& Estabrooks, C. (2003). The effects of hospital restructuring that included layoffs on individual nurses who remained employed: A systematic review of impact. International Journal of Sociology and Social Policy, 23(8/9), 8-53.

Emery, J.E. (1993). Perceived sources of stress among pediatric oncology nurses. Journal of Pediatric Oncology Nursing, 10(3), 87-92.

Escot, C., Artero, S., Gandubert, C., Boulenger, J.P., \& Ritchie, K. (2001). Stress levels in nursing staff working in oncology. Stress and Health, 17, 273-279. 
Fetterman, D.M. (1998). Ethnography (2nd ed.). Thousand Oaks, CA: Sage Publications.

Florio, G.A., Donnelly, J.P., \& Zevon, M.A. (1998). The structure of work-related stress and coping among oncology nurses in highstress medical settings: A transactional analysis. Journal of Occupational Health Psychology, 3(3), 227-242.

Gambles, M., Wilkinson, S.M., \& Dissanayake, C. (2003). What are you like? A personality profile of cancer and palliative care nurses in the United Kingdom. Cancer Nursing, 26(2), 97-104.

Garret, D.K., \& McDaniel, M.M. (2001). A new look at nurse burnout: The effects of environmental uncertainty and social climate. Journal of Nursing Administration, 31, 91-96.

Grunfeld, E., Whelan, T., Zitzelsberger, L., Willan, A., Montesanto, B., \& Evans, W.K. (2000). Cancer care workers in Ontario: Prevalence of burn-out, job stress and job satisfaction. Canadian Medical Association Journal, 163(2), 166-169.

Grunfeld, E., Zitzelsberger, L., Coristine, M., Whelan, T.J., Aspelund, F., \& Evans, W.K. (2005). Job stress and job satisfaction of cancer care workers. Psycho-oncology, 14, 61-69.

Haberman, M.R., Germino, B.B., Maliski, S., Stafford-Fox, V., \& Rice, K. (1994). What makes oncology nursing special? Walking the road together. Oncology Nursing Forum, 21(8), 41-47.

Hinds, P.S. (2000). Testing the stress-response sequence in pediatric oncology nursing. Journal of Pediatric Oncology Nursing, 17(2), 59-69.

Hinds, P.S., Fairclough, D.C., Dobos, C.L., Greer, R.H., Herring, P.L., Mayhill, J., et al. (1990). Development and testing of the stressor scale for pediatric oncology nurses. Cancer Nursing, 13(6), 35460.

Hinds, P.S., Quargnenti, A.G., Hickey, S.S., \& Mangum, G.H. (1994). A comparison of the stress-response sequence in new and experienced pediatric oncology nurses. Cancer Nursing, 17(1), 61-71.

Institut national du cancer du Canada. (2002). Statistiques canadiennes sur le cancer 2002. Toronto, ON.

Jenkins, J.F., \& Ostchega, Y. (1986). Evaluation of burn-out in oncology nurses. Cancer Nursing, 9(3), 108-116.

Kushnir, T., Rabin, S., \& Azulai, S. (1997). A descriptive study of stress management in a group of pediatric oncology nurses. Cancer Nursing, 20(6), 414-421.

Lamkin, L., Rosiak, J., Buerhaus, P., Mallory, G., \& Williams, M. (2002a). Oncology Nursing Society workforce survey. Part I: Perceptions of the nursing workforce environment and adequacy of nurse staffing in outpatient and inpatient oncology settings. Oncology Nursing Forum, 28(10), 1545-1115.

Lamkin, L., Rosiak, J., Buerhaus, P., Mallory, G., \& Williams, M. (2002b). Oncology Nursing Society workforce survey. Part II: Perceptions of the nursing workforce environment and adequacy of nurse staffing in outpatient and inpatient oncology settings. Oncology Nursing Forum, 29(1), 93-100.

Larson, D.G. (1992). The challenge of caring in oncology nursing. Oncology Nursing Forum, 19(6), 857-861.

Laschinger, H.K.S., Sabiston, J.A., Finegan, J., \& Shamian, J. (2001). Voices from the trenches: Nurses' experiences of hospital restructuring in Ontario [Electronic version]. Canadian Journal of Nursing Leadership, 14(1).

Laschinger, H.K.S., Shamian, J., Thomson, D. (2001). Impact of magnet hospital characteristics on nurses' perceptions of trust, burn-out, quality of care, and work satisfaction. Nursing Economics, 19(5), 209-219.

Leiter, M.P. (1991). The dream denied: Professional burn-out and the constraints of service organizations. Canadian Psychology, 32, 547-558.

Leiter, M.P., Harvie, P., \& Frizzell, C. (1998). The correspondence of patient satisfaction and nurse burn-out. Social Science and Medicine, 47, 1611-1617.
Leveck, M.L., \& Jones, C.B. (1996). The nursing practice environment, staff retention, and quality of care. Research in Nursing and Health, 19, 331-343.

Lewis, A.E. (1999). Reducing burn-out: Development of an oncology staff bereavement program. Oncology Nursing Forum 26(6), 1065-1069.

Luborsky, I.M. (1994). The identification and analysis of themes and patterns. In J.F. Gubrium \& A. Sankar (Eds.). Qualitative methods in aging research (pp. 189-210). Thousand Oaks, CA: Sage Publications.

Maslach, C. (1976). Burned-out. Human Behavior, 5(9), 16-22.

Maslach, C., \& Leiter, M. (1998). The truth about burn-out: How organizations cause personal stress and what to do about it. San Francisco: Jossey-Bass.

Maslach, C., Schaufeli, W.B., \& Leiter, M.P. (2001). Job burn-out. Annual Review of Psychology, 52, 397-422.

Maurier, W.L., \& Northcott, H.D. (2000). Job uncertainty and health status for nurses during restructuring of health care in Alberta. Western Journal of Nursing Research, 22(5), 623-41.

McElroy, A.M. (1982). Burn-out: A review of the literature with application to cancer nursing. Cancer Nursing, 5(3), 211-217.

Medland, J., Howard-Ruben, J., \& Whitaker, E. (2004). Fostering psychosocial wellness in oncology nurses: Addressing burn-out and social support in the workplace. Oncology Nursing Forum, 31(1), 47-54.

Morse, J.M., \& Field, P.A. (1995). Qualitative research methods for health professionals (2nd ed.). Thousand Oaks, CA: Sage Publications.

Morse, J.M., \& Richards, L. (2002). ReadMe first for a user's guide to qualitative methods. Thousand Oaks, CA: Sage Publications.

Oehler, S., Davidson, M., Starr, L., \& Lee, D. (1991). Burn-out, job stress, anxiety and perceived social support in neonatal nurses. Heart and Lung, 2, 500-505.

Papadatou, D., Anagnostopoulos, F., \& Monos, D. (1994). Factors contributing to the development of burn-out in oncology nursing. British Journal of Medical Psychology, 67, 187-199.

Penson, R., Dignan, F., Canellos, G., Picard, C., \& Lynch, T. (2000). Burn-out: Caring for the caregivers. Oncologist, 5, 425-434.

Pines, A., \& Kranner, A.D. (1982). Nurse's burn-out: Lack of positive conditions and presence of negative conditions as two independent sources of stress. Journal of Psychological Nursing and Mental Health Services, 20(8), 30-35.

Porter-O'Grady, T., \& Malloch, K. (2003). Quantum leadership: A textbook of new leadership. Sudbury, MA: Jones and Bartlett Publishers.

Robinson, S., Roth, S., Keim J., Levenson, M., Flentje, J., \& Bashor, K. (1991). Nurse burn-out: Work-related and demographic factors as culprits. Research in Nursing and Health, 14, 223-228.

Roper, J.M., \& Shapira, J. (2000). Ethnography in nursing research. Thousand Oaks, CA: Sage Publications.

Spradley, J.P. (1979). The ethnographic interview. New York: Holt, Rinehart and Winston.

Steen, B., Burghen, E., Hinds, P.S., Srivastava, D.K., \& Tong, X. (2003). Development and testing of the role-related meaning scale for staff in pediatric oncology. Cancer Nursing, 26(3), 187-194.

Steeves, R., Cohen, M.Z., \& Wise, C.T. (1994). An analysis of critical incidents describing the essence of oncology nursing. Oncology Nursing Forum, 21(8), 19-25.

Stewart, B.E., Meyerowitz, B.E., Jackson, L.E., Yarkin, K.L., \& Harvey, J. H. (1982). Psychological stress associated with outpatient oncology nursing. Cancer Nursing, 5(5), 383-387.

Yasko, J.M. (1983). Variables which predict burn-out experienced by oncology clinical nurse specialists. Cancer Nursing, 6, 109-116.

Wilkinson, S.M. (1994). Stress in cancer nursing: Does it really exist? Journal of Advanced Nursing, 20, 1079-1084. 